\title{
VERITAS SEARCH FOR VHE GAMMA-RAY EMISSION FROM DWARF SPHEROIDAL GALAXIES
}

V. A. Acciari ${ }^{1}$, T. Arlen ${ }^{2}$, T. Aune ${ }^{3}$, M. Beilicke ${ }^{4}$, W. Benbow ${ }^{1}$, D. Boltuch ${ }^{5}$, S. M. Bradbury ${ }^{6}$, J. H. Buckley ${ }^{4}$,

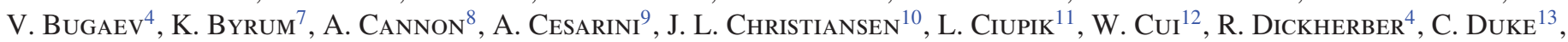
J. P. Finle ${ }^{12}$, G. Finnegan ${ }^{14}$, A. Furniss ${ }^{3}$, N. Galante ${ }^{1}$, S. Godambe ${ }^{14}$, J. Grube ${ }^{11}$, R. Guenette ${ }^{15}$, G. GyuK ${ }^{11}$, D. Hanna ${ }^{15}$,

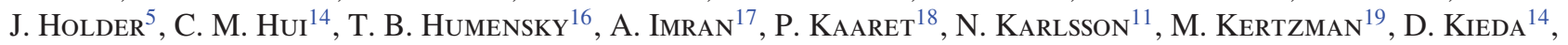
A. Konopelko $^{20}$, H. KrawCZYNSKi ${ }^{4}$, F. Krennrich ${ }^{17}$, G. Maier ${ }^{15,25}$, S. McArThur ${ }^{4}$, A. McCanN ${ }^{15}$, M. McCutcheON ${ }^{15}$, P. Moriarty ${ }^{21}$, R. A. $\mathrm{Ong}^{2}$, A. N. Otte ${ }^{3}$, D. Pandel ${ }^{18}$, J. S. Perkins ${ }^{1}$, M. Pohl ${ }^{17,26}$, J. Quinn ${ }^{8}$, K. Ragan ${ }^{15}$, L. C. Reyes ${ }^{22}$, P. T. Reynolds ${ }^{23}$, E. Roache ${ }^{1}$, H. J. Rose ${ }^{6}$, M. Schroedter ${ }^{17}$, G. H. Sembroski ${ }^{12}$, G. Demet Senturk ${ }^{24}$, A. W. Smith ${ }^{7}$, D. Steele ${ }^{11,27}$, S. P. Swordy ${ }^{16}$, G. Teší́ ${ }^{15}$, M. Theiling ${ }^{1}$, S. Thibadeau ${ }^{4}$, A. Varlotta ${ }^{12}$, V. V. Vassiliev ${ }^{2}$, S. Vincent $^{14}$, R. G. Wagner ${ }^{7}$, S. P. Wakely ${ }^{16}$, J. E. Ward ${ }^{8}$, T. C. Weekes ${ }^{1}$, A. Weinstein ${ }^{2}$, T. Weisgarber ${ }^{16}$, D. A. Williams ${ }^{3}$, S. Wissel $^{16}$, AND B. ZITZER ${ }^{12}$

\author{
${ }^{1}$ Fred Lawrence Whipple Observatory, Harvard-Smithsonian Center for Astrophysics, Amado, AZ 85645, USA \\ ${ }^{2}$ Department of Physics and Astronomy, University of California, Los Angeles, CA 90095, USA \\ ${ }^{3}$ Santa Cruz Institute for Particle Physics and Department of Physics, University of California, Santa Cruz, CA 95064, USA \\ ${ }^{4}$ Department of Physics, Washington University, St. Louis, MO 63130, USA \\ ${ }^{5}$ Department of Physics and Astronomy and the Bartol Research Institute, University of Delaware, Newark, DE 19716, USA \\ ${ }^{6}$ School of Physics and Astronomy, University of Leeds, Leeds, LS2 9JT, UK \\ ${ }^{7}$ Argonne National Laboratory, 9700 S. Cass Avenue, Argonne, IL 60439, USA; rgwcdf@hep.anl.gov \\ ${ }^{8}$ School of Physics, University College Dublin, Belfield, Dublin 4, Ireland \\ ${ }^{9}$ School of Physics, National University of Ireland Galway, University Road, Galway, Ireland \\ ${ }^{10}$ Physics Department, California Polytechnic State University, San Luis Obispo, CA 94307, USA \\ ${ }^{11}$ Astronomy Department, Adler Planetarium and Astronomy Museum, Chicago, IL 60605, USA \\ 12 Department of Physics, Purdue University, West Lafayette, IN 47907, USA \\ ${ }^{13}$ Department of Physics, Grinnell College, Grinnell, IA 50112-1690, USA \\ ${ }^{14}$ Department of Physics and Astronomy, University of Utah, Salt Lake City, UT 84112, USA \\ ${ }^{15}$ Physics Department, McGill University, Montreal, QC H3A 2T8, Canada \\ ${ }^{16}$ Enrico Fermi Institute, University of Chicago, Chicago, IL 60637, USA \\ ${ }^{17}$ Department of Physics and Astronomy, Iowa State University, Ames, IA 50011, USA \\ ${ }^{18}$ Department of Physics and Astronomy, University of Iowa, Van Allen Hall, Iowa City, IA 52242, USA \\ ${ }_{19}$ Department of Physics and Astronomy, DePauw University, Greencastle, IN 46135-0037, USA \\ ${ }^{20}$ Department of Physics, Pittsburg State University, 1701 South Broadway, Pittsburg, KS 66762, USA \\ ${ }^{21}$ Department of Life and Physical Sciences, Galway-Mayo Institute of Technology, Dublin Road, Galway, Ireland \\ ${ }^{22}$ Kavli Institute for Cosmological Physics, University of Chicago, Chicago, IL 60637, USA \\ ${ }^{23}$ Department of Applied Physics and Instrumentation, Cork Institute of Technology, Bishopstown, Cork, Ireland \\ ${ }^{24}$ Columbia Astrophysics Laboratory, Columbia University, New York, NY 10027, USA \\ Received 2010 April 22; accepted 2010 June 28; published 2010 August 18
}

\begin{abstract}
Indirect dark matter searches with ground-based gamma-ray observatories provide an alternative for identifying the particle nature of dark matter that is complementary to that of direct search or accelerator production experiments. We present the results of observations of the dwarf spheroidal galaxies Draco, Ursa Minor, Boötes 1, and Willman 1 conducted by the Very Energetic Radiation Imaging Telescope Array System (VERITAS). These galaxies are nearby dark matter dominated objects located at a typical distance of several tens of kiloparsecs for which there are good measurements of the dark matter density profile from stellar velocity measurements. Since the conventional astrophysical background of very high energy gamma rays from these objects appears to be negligible, they are good targets to search for the secondary gamma-ray photons produced by interacting or decaying dark matter particles. No significant gamma-ray flux above $200 \mathrm{GeV}$ was detected from these four dwarf galaxies for a typical exposure of $\sim 20 \mathrm{hr}$. The $95 \%$ confidence upper limits on the integral gamma-ray flux are in the range (0.4-2.2) $\times 10^{-12}$ photons $\mathrm{cm}^{-2} \mathrm{~s}^{-1}$. We interpret this limiting flux in the context of pair annihilation of weakly interacting massive particles (WIMPs) and derive constraints on the thermally averaged product of the total self-annihilation cross section and the relative velocity of the WIMPs $\left(\langle\sigma v\rangle \lesssim 10^{-23} \mathrm{~cm}^{3} \mathrm{~s}^{-1}\right.$ for $m_{\chi} \gtrsim 300 \mathrm{GeV} \mathrm{c}^{-2}$ ). This limit is obtained under conservative assumptions regarding the dark matter distribution in dwarf galaxies and is approximately 3 orders of magnitude above the generic theoretical prediction for WIMPs in the minimal supersymmetric standard model framework. However, significant uncertainty exists in the dark matter distribution as well as the neutralino cross sections which under favorable assumptions could further lower this limit.
\end{abstract}

Key words: dark matter - galaxies: dwarf - gamma rays: galaxies

Online-only material: color figure

\footnotetext{
${ }^{25}$ Now at DESY, Platanenallee 6, 15738 Zeuthen, Germany.

${ }^{26}$ Now at Institut für Physik und Astronomie, Universität Potsdam, 14476 Potsdam-Golm, Germany; DESY, Platanenallee 6, 15738 Zeuthen, Germany.

${ }^{27}$ Now at Los Alamos National Laboratory, MS H803, Los Alamos, NM 87545.
}

\section{INTRODUCTION}

The existence of astrophysical non-baryonic dark matter (DM) has been established by its gravitational effects on a wide range of spatial scales. Perhaps the most compelling evidence for the existence of weakly interacting particle dark matter comes 
from observations of colliding galaxy clusters in which the baryonic matter in the form of X-ray emitting gas is separated from the source of the gravitational potential detected through gravitational lensing (Clowe et al. 2006; Bradač et al. 2008). However, despite the well-established presence of DM in the universe, its particle nature is unknown.

The quest to understand the nature of DM draws upon research in cosmology, particle physics, and astroparticle physics with direct and indirect detection experiments (Bergström 2000; Bertone et al. 2005). In this paper, we focus on the indirect search for very high energy (VHE; energy > $100 \mathrm{GeV}$ ) gamma rays resulting from the interaction or decay of DM particles in astrophysical objects in which the gravitational potential is dominated by DM.

Among many theoretical candidates for the DM particle (Taoso et al. 2008), a weakly interacting massive particle (WIMP) is among the best-motivated ones. A thermal relic of the early universe with an interaction cross section on the weak scale will naturally produce the present-day DM density if the particle has a weak-scale mass (Lee \& Weinberg 1977; Dicus et al. 1977; $\Omega_{\mathrm{DM}} h^{2}=0.1099 \pm 0.0062$ (WMAP only), $\Omega_{\mathrm{DM}} h^{2}=0.1131 \pm 0.0034$ (WMAP + Baryon Acoustic Oscillations + Type Ia Supernovae), where $\Omega_{\mathrm{DM}}$ is the ratio of dark matter density to the critical density for a flat universe and $h$ is a dimensionless quantity defined as the Hubble constant, $H_{\circ}$, normalized to $100 \mathrm{~km} \mathrm{~s}^{-1} \mathrm{Mpc}^{-1}$ (Komatsu et al. 2009)). Several candidates for WIMPs are predicted in extensions to the standard model of particle physics, for example, the neutralino from supersymmetry (Ellis et al. 1984) and the Kaluza-Klein particle in theories of universal extra dimensions (Servant \& Tait 2003; Bertone et al. 2003). Both neutralinos and Kaluza-Klein particles are predicted to have a mass in the range of a few tens of $\mathrm{GeV} c^{-2}$ to possibly a few $\mathrm{TeV} c^{-2}$.

The self-annihilation of WIMPs produces a unique spectral signature of secondary gamma rays which is expected to significantly deviate from the standard power-law behavior observed in most conventional astrophysical sources of VHE gamma rays and would have a cutoff at the WIMP mass. In addition, it could exhibit a monoenergetic line at the WIMP mass or a considerable enhancement of gamma-ray photons at the endpoint of the spectrum due to the internal bremsstrahlung effect (Bringmann et al. 2008). Observation of these spectral signatures combined with the spatial distribution of the gammaray flux from an astrophysical source is a unique capability of indirect DM searches utilizing gamma rays.

Nearby astrophysical objects with the highest dark matter density are natural candidates for indirect DM searches. While the Galactic Center is likely to be the brightest source of annihilation radiation (e.g., see Bergström et al. 1998), VHE gamma-ray measurements reveal a bright gamma-ray source at the center which constitutes a large astrophysical background (Aharonian et al. 2006a). Other possible bright sources are expected to be the cores of nearby large galaxies such as M31 or halos around galactic intermediate mass black holes, should they exist, where adiabatic compression of dark matter halos could result in a large enhancement in the annihilation signal, in some cases already exceeding experimental bounds (Bringmann et al. 2009b; Bertone et al. 2009). However, in these regions, the DM density profiles are poorly constrained and, in the case of nearby galaxies, conventional astrophysical VHE sources can generate backgrounds for DM annihilation searches. In contrast, the satellite dwarf spheroidal galaxies (dSphs) of the Milky Way are attractive targets for indirect dark matter searches due to their proximity (20-100 kpc) and relatively well-constrained DM profiles derived from stellar kinematics. They, in fact, may be the brightest sources for annihilation radiation after the Galactic Center (Bullock et al. 2009). The general lack of active or even recent star formation in most $\mathrm{dSphs}$ implies that there is little background from conventional astrophysical VHE processes as has been observed in the Milky Way Galactic Center (Kosack et al. 2004; Aharonian et al. 2006b, 2009). The growing class of nearby dSphs discovered by recent all-sky surveys (York et al. 2000; Belokurov et al. 2007) increases the probability of finding an object for which the halo density is sufficient to yield a detectable gamma-ray signal.

In this paper, we report on an indirect DM search for gamma rays from four dSphs: Draco, Ursa Minor, Boötes 1, and Willman 1, carried out using the Very Energetic Radiation Imaging Telescope Array System (VERITAS). After a brief summary of the properties of the observational targets and previous VHE observations in Section 2, we describe the VERITAS instrument, the data set, and the analysis techniques in Section 3. Sections 4 and 5 are devoted to the discussion of the results and their interpretation in terms of constraints on the WIMP parameter space. We conclude in Section 6 with a discussion of the opportunities for indirect DM detection by future ground-based gamma-ray instrumentation.

\section{OBSERVATIONAL TARGETS}

Three of the dSphs forming the subject of this paper, Draco, Ursa Minor, and Willman 1, have been identified as the objects within the dSph class with potentially the highest gamma-ray self-annihilation flux, e.g., see Strigari et al. (2007, 2008). The modeling of the DM distribution of these galaxies usually is based on stellar kinematics assuming a spherically symmetric stellar population and an Navarro-Frenk-White (NFW) profile for DM (Navarro et al. 1997) characterized by two parameters: the scale radius $r_{s}$ and scale density $\rho_{s}$,

$$
\rho(r)=\rho_{s}\left(\frac{r}{r_{s}}\right)^{-1}\left(1+\frac{r}{r_{s}}\right)^{-2} .
$$

The properties of these galaxies including constraints on $r_{s}$ and $\rho_{s}$ as found in Strigari et al. (2007) and Strigari et al. (2008) are summarized in Table 1.

The Draco dSph is one of the most frequently studied objects for indirect DM detection (Baltz et al. 2000; Tyler 2002; Evans et al. 2004; Colafrancesco et al. 2007; Sánchez-Conde et al. 2007; Strigari et al. 2007, 2008; Bringmann et al. 2009a). It has an approximately spherically symmetric stellar distribution (Irwin \& Hatzidimitriou 1995) with total luminosity of the order of $10^{5} L_{\odot}$ (Piatek et al. 2002). The large spectroscopic data set available for this object (Wilkinson et al. 2004; Muñoz et al. 2005; Walker et al. 2007) tightly constrains its DM distribution profile. Draco is consistent with an old low-metallicity $([\mathrm{Fe} / \mathrm{H}]=-1.8 \pm 0.2)$ stellar population with no significant star formation over the last 2 Gyr (Aparicio et al. 2001). Draco previously has been observed at VHE energies by the STACEE observatory (Driscoll et al. 2008), the Whipple $10 \mathrm{~m}$ telescope (Wood et al. 2008), and the MAGIC telescope (Albert et al. 2008).

The Ursa Minor dSph has a distance and inferred DM content similar to those of Draco. There is no evidence of young or intermediate-age stellar populations in Ursa Minor (Shetrone et al. 2001). Photometric studies of this object have found 
Table 1

Properties of the Four dSphs

\begin{tabular}{lcccc}
\hline \hline \multicolumn{1}{c}{ Quantity } & Draco & Ursa Minor & Boötes 1 & Willman 1 \\
\hline$\alpha[\mathrm{J} 2000.0]$ & $17^{\mathrm{h}} 20^{\mathrm{m}} 12^{\mathrm{s}} .4$ & $15^{\mathrm{h}} 09^{\mathrm{m}} 11^{\mathrm{s}} .3$ & $14^{\mathrm{h}} 00^{\mathrm{m}} 06^{\mathrm{s}}$ & $10^{\mathrm{h}} 49^{\mathrm{m}} 22^{\mathrm{s}} .3$ \\
$\delta[\mathrm{J} 2000.0]$ & $57^{\circ} 54^{\prime} 55^{\prime \prime}$ & $67^{\circ} 12^{\prime} 52^{\prime \prime}$ & $14^{\circ} 30^{\prime} 00^{\prime \prime}$ & $51^{\circ} 03^{\prime} 03^{\prime \prime}$ \\
$L_{V}\left[\mathrm{~L}_{\odot}\right]$ & $(2.7 \pm 0.4) \times 10^{5}$ & $(2.0 \pm 0.9) \times 10^{5}$ & $(3.0 \pm 0.6) \times 10^{4}$ & $(1.0 \pm 0.7) \times 10^{3}$ \\
$r_{h}[\mathrm{pc}]$ & $221 \pm 16$ & $150 \pm 18$ & $242 \pm 21$ & $25 \pm 6$ \\
$R_{d}[\mathrm{kpc}]$ & 80 & 66 & 62 & 38 \\
$\rho_{s}\left[\mathrm{M}_{\odot} / \mathrm{kpc}^{3}\right]$ & $4.5 \times 10^{7}$ & $4.5 \times 10^{7}$ & $\ldots$ & $4 \times 10^{8}$ \\
$r_{s}[\mathrm{kpc}]$ & 0.79 & 0.79 & 3 & 0.18 \\
$J\left(\rho_{s}, r_{s}\right)$ & 4 & 7 & 3 & 22 \\
\hline
\end{tabular}

Notes. Preferred values for DM halo parameters, $\rho_{s}$ and $r_{s}$, which are defined in the text, are taken from Strigari et al. (2007) and Bringmann et al. (2009a). Values for $L_{V}$, the visual luminosity, and $r_{h}$, the half-light radius are taken from Walker et al. (2009). $R_{d}$ is the heliocentric distance of the $\mathrm{dSph}$. The calculation of the dimensionless line-of-sight integral, $J$, which is normalized to the critical density squared times the Hubble radius $\left(3.832 \times 10^{17} \mathrm{GeV}^{2} \mathrm{~cm}^{-5}\right)$, is explained in Section 5. The $J$ value for Boötes was calculated by G. D. Martinez and J. S. Bullock. As explained in the text, the elongation of Boötes and the relative lack of stellar kinematic data lead to large uncertainties for $r_{s}$ or $\rho_{s}$ and no values are provided in this case.

evidence for significant structures in the stellar distribution in the central 10' (Bellazzini et al. 2002; Kleyna et al. 2003) and an extratidal stellar population (Palma et al. 2003). These unusual morphological characteristics could be evidence of possible tidal interaction with the Milky Way, velocity projection effects along the line of sight, or the presence of fluctuations in the DM induced gravitational potential (Kleyna et al. 2003). In fact, such confusing factors are present in most dSph galaxies. Ursa Minor was previously studied at VHE energies by the Whipple $10 \mathrm{~m}$ telescope (Wood et al. 2008).

The recently discovered dSph Boötes 1 (Belokurov et al. 2006) shows evidence for elongation of the stellar profile. $\mathrm{N}$-body simulations cannot reproduce the observed velocity dispersion without a dominant contribution from DM. In addition, modeling of the tidal interaction effects between Boötes 1 and the Milky Way does not provide an adequate explanation for the elongation of this system, suggesting a non-spherically symmetric distribution of DM in the Boötes progenitor (Fellhauer et al. 2008). Given that the stellar kinematical data are based on about 30 stars, the scale radius and density of the NFW profile have large uncertainty as well as significant degeneracy. Thus, values for $r_{s}$ and $\rho_{s}$ are unavailable in the literature. The modeling of Boötes 1 was done by G. D. Martinez and J. S. Bullock (2009, private communication) for a range of NFW fits. The methodology is described in Martinez et al. (2009) and Abdo et al. (2010). They produce a probability density function (pdf) for $J$, the astrophysical contribution to the flux (see Section 5), which is approximately Gaussian in $\log (J)$. The value given in Table 1 represents $J$ at the peak of the pdf which is approximately the mean of the distribution. The estimates of the age of the stellar population and metallicity suggest a similarity with the old and metal-poor $([\mathrm{Fe} / \mathrm{H}] \sim-2.5$ to -2.1$)$ stellar distribution of M92 (Belokurov et al. 2006; Muñoz et al. 2006; Martin et al. 2007). To date, no other VHE gamma-ray observations have been reported for this object.

Together with Boötes 1, Willman 1 belongs to the new class of low surface brightness dSphs recently discovered by the Sloan Digital Sky Survey (Willman et al. 2005). Willman 1 is one of the smallest $\left(r_{h} \sim 25 \mathrm{pc}\right)$ and least luminous $\left(L_{V} \sim 10^{3} L_{\odot}\right)$ $\mathrm{dSphs}$ known. Its half-light radius and absolute magnitude suggest that it may be an intermediate object between dwarf galaxies and globular clusters (Belokurov et al. 2007). Due to the small kinematic sample of stars available for this object, the constraints on the DM halo parameterization are poor (Strigari et al. 2008). Latest estimates of the metallicity suggest a low value of $[\mathrm{Fe} / \mathrm{H}]$ which is consistent with the observed trend of decreasing metallicity for fainter dSphs (Siegel et al. 2008). The MAGIC collaboration has recently reported the results of the observations of Willman 1 (Aliu et al. 2009).

\section{DATA AND ANALYSIS}

\subsection{The VERITAS Observatory}

The VERITAS observatory is an array of four $12 \mathrm{~m}$ imaging atmospheric Cherenkov telescopes (IACTs) located at the Fred Lawrence Whipple Observatory $\left(31^{\circ} 57^{\prime} \mathrm{N} 111^{\circ} 37^{\prime} \mathrm{W}\right)$ in southern Arizona at an altitude of $1.27 \mathrm{~km}$ above sea level (Weekes et al. 2002). The observatory is sensitive over an energy range of $150 \mathrm{GeV}$ to $30 \mathrm{TeV}$ with an energy resolution of 10\%-20\% and an angular resolution (68\% containment) of $<0.14$ per event. For the measurements reported here, VERITAS had a point source sensitivity capable of detecting gamma rays with a flux of $5 \%(1 \%)$ of the Crab Nebula flux above $300 \mathrm{GeV}$ at five standard deviations in $<2.5(<50) \mathrm{hr}$ at $20^{\circ}$ zenith angle. During summer 2009, subsequent to the four dSph observations, the array configuration was changed, improving the point source sensitivity. Further technical description of the VERITAS observatory can be found in Acciari et al. (2008).

\subsection{Data}

Observations of the Draco, Ursa Minor, Boötes 1, and Willman 1 dSphs were performed during 2007-2009 (see Table 2). Observations were taken in "wobble" mode (Berge et al. 2007) with the source offset by 0.5 from the center of the field of view in order to obtain the source and background measurement within the same observation. The direction of the offset was alternated between north, south, east, and west to minimize systematic errors. Reflected background regions are defined within the field of view at the same radius with respect to the camera center as that of the targeted dwarf galaxy. Observations were made with varying atmospheric conditions during moonless periods of the night. Data were quality selected for analysis based on the stability of the cosmic-ray trigger rate and the rms temperature fluctuations observed by an FIR camera viewing the sky in the vicinity of the observed target $\left(\leqslant 0.3^{\circ} \mathrm{C}\right)$. The total exposure on each source is given in Table 2 . 
Table 2

Summary of Observation Periods and Exposures of dSphs by VERITAS

\begin{tabular}{llcc}
\hline \hline \multicolumn{1}{c}{ Source } & \multicolumn{1}{c}{ Period } & Exposure $(\mathrm{hr})$ & Zenith Angle $\left(^{\circ}\right)$ \\
\hline Draco & 2007 Apr-May & 18.38 & $26-51$ \\
Ursa Minor & 2007 Feb-May & 18.91 & $35-46$ \\
Boötes 1 & 2009 Apr-May & 14.31 & $17-29$ \\
Willman 1 & 2007 Dec-2008 Feb & 13.68 & $19-28$ \\
\hline
\end{tabular}

\subsection{Analysis}

Data reduction follows the methods described in Acciari et al. (2008). A brief outline of the analysis flow follows. Images recorded by each of the VERITAS telescopes are characterized by a second moment analysis giving the Hillas parameters (Hillas 1985). A stereoscopic analysis of the image parameters is used to reconstruct the gamma-ray arrival direction and shower core position. The background of cosmic rays is reduced by a factor of $>10^{5}$ utilizing cuts on the reconstructed arrival direction $\left(\theta^{2}<0.013 \mathrm{deg}^{2}\right)$ and the image shape parameters, mean scaled width and length $(0.05$ msw 1.16 and 0.05 msl 1.36). The image distance from the center of the camera is required to be less than 1.43 to avoid truncation effects at the edge of the 3.5 field of view. The integrated charge recorded in at least two telescopes is further required to be $>75$ photoelectrons (400 digital counts) which effectively sets the energy threshold of the analysis to be above $\sim 200 \mathrm{GeV}$ depending on the zenith angle. The energy threshold quoted in our analyses is taken to be the energy at which the differential detection rate of gamma rays from the Crab Nebula peaks. The cuts applied in this analysis were optimized to maximize significance of the detection for a hypothetical source with a power-law spectrum $(d F / d E=3.2 \times$ $\left.10^{-12}(E / \mathrm{TeV})^{-2.5} \mathrm{~cm}^{-2} \mathrm{~s}^{-1} \mathrm{TeV}^{-1}\right)$ corresponding to $3 \%$ of the Crab Nebula flux. Two independent data analysis packages were used to analyze the data and yielded consistent results.

The significance of the detection was calculated by comparing the counts in the source region to the expected background counts. The background in the source region is estimated using the reflected region model. In this model circular background regions, here of angular radius 0.115 , are defined with an offset from the camera center equal to that of the putative source. Eleven background regions can be accommodated within the VERITAS field of view. The absence of bright stars within any of the four dSph pointings allows all 11 regions to be used in the background count estimation. The significance of any signal is computed using the Li and Ma method (Li \& Ma 1983, Equation (17)).

\section{RESULTS}

Table 3 summarizes the results for each of the four dSphs. The effective energy threshold for each of the targets changes primarily due to the average zenith angle of observations. The table shows the average effective collecting area for gamma rays as calculated from a sample of simulated gamma-ray showers. No significant excesses of counts above background were detected from these observations. The $95 \%$ confidence level upper limits on the gamma-ray integral flux were calculated using the bounded profile likelihood ratio statistic developed by Rolke et al. (2005).

As we have noted, Draco, Ursa Minor, and Willman I have been observed by other IACTs and we briefly compare our flux limits to the other observations. For Draco, STACEE (Driscoll et al. 2008) finds a spectral limit of less than $1.6 \times 10^{-13}\left(\frac{E}{200 \mathrm{GeV}}\right)^{-2.2} \mathrm{~cm}^{-2} \mathrm{~s}^{-1} \mathrm{GeV}^{-1}$. The MAGIC flux limit (Albert et al. 2008) from observation of Draco is $1.1 \times 10^{-11} \mathrm{~cm}^{-2} \mathrm{~s}^{-1}$ above a threshold of $140 \mathrm{GeV}$. MAGIC also has set flux limits for Willman 1 in the range (5.7-9.9) $\times$ $10^{-12} \mathrm{~cm}^{-2} \mathrm{~s}^{-1}$ above $100 \mathrm{GeV}$ based on several benchmark models (Aliu et al. 2009), compared to our limit of $1.17 \times$ $10^{-12} \mathrm{~cm}^{-2} \mathrm{~s}^{-1}$ above a threshold of $320 \mathrm{GeV}$. The limits for the VERITAS observations of Draco and Ursa Minor are an improvement of about a factor of 40 over the earlier observations of the group on the Whipple $10 \mathrm{~m}$ IACT (Wood et al. 2008).

Figure 1 shows the upper limits on the differential spectral energy density $\left(E^{2} d \phi / d E\right)$ as a function of energy. The upper limits were derived with four equidistant log energy bins per decade requiring $95 \%$ CL in each bin.

\section{LIMITS ON WIMP PARAMETER SPACE}

The differential flux of gamma rays from WIMP selfannihilation is given by

$$
\frac{d \phi(\Delta \Omega)}{d E}=\frac{\langle\sigma v\rangle}{8 \pi m_{\chi}^{2}}\left[\frac{d N\left(E, m_{\chi}\right)}{d E}\right] \int_{\Delta \Omega} d \Omega \int \rho^{2}(\lambda, \Omega) d \lambda,
$$

where $\langle\sigma v\rangle$ is the thermally averaged product of the total selfannihilation cross section and the velocity of the WIMP, $m_{\chi}$ is the WIMP mass, $d N\left(E, m_{\chi}\right) / d E$ is the differential gamma-ray yield per annihilation, $\Delta \Omega$ is the observed solid angle around

Table 3

Results of Observations of dSphs by VERITAS

\begin{tabular}{lcccc}
\hline \hline \multicolumn{1}{c}{ Quantity } & Draco & Ursa Minor & Boötes 1 & Willman 1 \\
\hline Exposure (s) & 66185 & 68080 & 51532 & 49255 \\
On source (counts) & 305 & 250 & 429 & 326 \\
Total background (counts) & 3667 & 11 & 4405 & 3602 \\
Number of background regions & 11 & -1.77 & 11 & 11 \\
Significance & -1.51 & 15.6 & 7.35 & -0.08 \\
95\% CL (counts) & 18.8 & $5.71 \times 10^{8}$ & $6.37 \times 10^{8}$ & 36.7 \\
Average effective area $\left(\mathrm{cm}^{2}\right)$ & 340 & 380 & 300 & $6.37 \times 10^{8}$ \\
Energy threshold $\left(\mathrm{GeV}^{\mathrm{c}}\right.$ & $0.49 \times 10^{-12}$ & $0.40 \times 10^{-12}$ & $2.19 \times 10^{-12}$ & $1.17 \times 10^{-12}$ \\
Flux limit 95\% CL $\left(\mathrm{cm}^{-2} \mathrm{~s}^{-1}\right)$ & & & & 320
\end{tabular}

Notes.

${ }^{\mathrm{a}} \mathrm{Li}$ and Ma method (Li \& Ma 1983).

${ }^{b}$ Rolke method (Rolke et al. 2005).

${ }^{\mathrm{c}}$ Definition given in the text. 


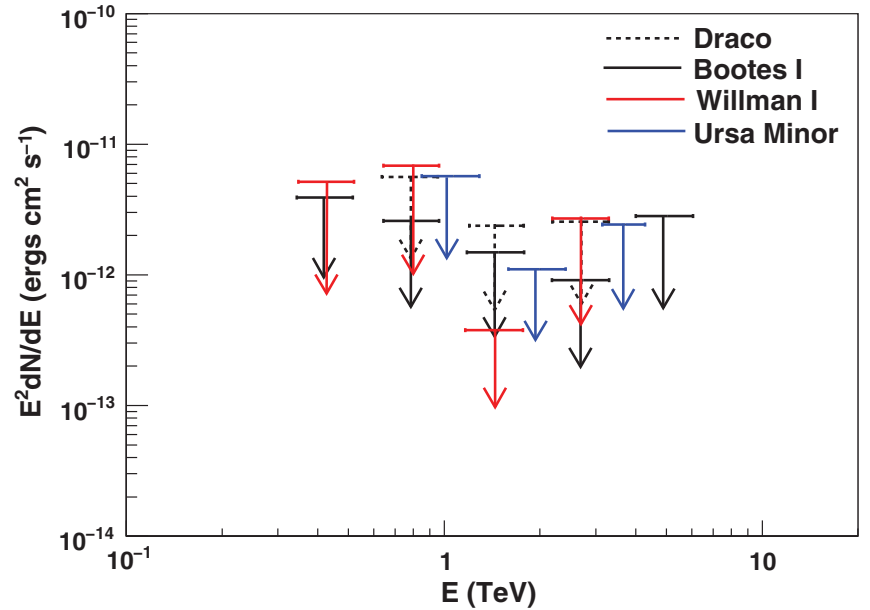

Figure 1. 95\% CL upper limits on the spectral energy density $\left(\mathrm{erg} \mathrm{cm}^{-2} \mathrm{~s}^{-1}\right)$ as a function of gamma-ray energy for the four dSphs.

(A color version of this figure is available in the online journal.)

the dwarf galaxy center, $\rho$ is the DM mass density, and $\lambda$ is the line-of-sight distance to the differential integration volume. The astrophysical contribution to the flux can be expressed by the dimensionless factor $J$

$$
J(\Delta \Omega)=\left(\frac{1}{\rho_{c}^{2} R_{H}}\right)_{\Delta \Omega} d \Omega \quad \rho^{2}(\lambda, \Omega) d \lambda,
$$

which has been normalized to the product of the square of the critical density, $\rho_{c}=9.74 \times 10^{-30} \mathrm{~g} \mathrm{~cm}^{-3}$ and the Hubble radius, $R_{H}=4.16 \mathrm{Gpc}$ following Wood et al. (2008).

Based on Equation (2), the upper limits on the gammaray rate, $R_{\gamma}(95 \% \mathrm{CL})$, constrain the WIMP parameter space $\left(m_{\chi},\langle\sigma v\rangle\right)$ according to

$$
\begin{gathered}
\frac{R_{\gamma}(95 \% \mathrm{C} L)}{\mathrm{hr}^{-1}}>\frac{J}{1.09 \times 10^{4}}\left(\frac{\langle\sigma v\rangle}{3 \times 10^{-26} \mathrm{~cm}^{3} \mathrm{~s}^{-1}}\right) \\
\times \infty \frac{A(E)}{5 \times 10^{8} \mathrm{~cm}^{2}}\left(\frac{300 \mathrm{GeV} \mathrm{c} c^{-2}}{m_{\chi}}\right)^{2} \\
\times \frac{E d N / d E\left(E, m_{\chi}\right)}{10^{-2}} \frac{d E}{E},
\end{gathered}
$$

where $A(E)$ is the energy-dependent gamma-ray collecting area. The expression has been cast as a product of dimensionless factors with the variables normalized to representative quantities, e.g., the cross section times velocity is normalized to $3 \times 10^{-26} \mathrm{~cm}^{3} \mathrm{~s}^{-1}$ which is a rough generic prediction for $\langle\sigma v\rangle$ for a WIMP thermal relic in the absence of coannihilations for $m_{\chi}>100 \mathrm{GeV} c^{-2}$ (cf. Figure 2). The main contribution to the integral comes from the energy range in the vicinity of the energy threshold ( $E \simeq 300 \mathrm{GeV}$ for observations in this paper) where $A(E)$ changes rapidly. For VERITAS the effective area at $300 \mathrm{GeV}$ is $\sim 6 \times 10^{8} \mathrm{~cm}^{2}$. For a representative MSSM model, $E d N / d E$ at $300 \mathrm{GeV}$ is a function of neutralino mass, $m_{\chi}$, and it changes in the range $10^{-2}-10^{-1}$ for $m_{\chi}$ from $300 \mathrm{GeV} c^{-2}$ to a few $\mathrm{TeV} c^{-2}$. Although $E d N / d E$ is a rapid function of $m_{\chi}$, this dependence is nearly compensated by the $\left(300 \mathrm{GeV} \mathrm{c} c^{-2} / m_{\chi}\right)^{2}$ prefactor. The product of these two contributions and, consequently, the overall integral value is weakly dependent on the neutralino mass within the indicated range and is on the order of 1. It is evident from the inequality (Equation (4)) that for a typical upper limit on the detection rate of 1 gamma ray per

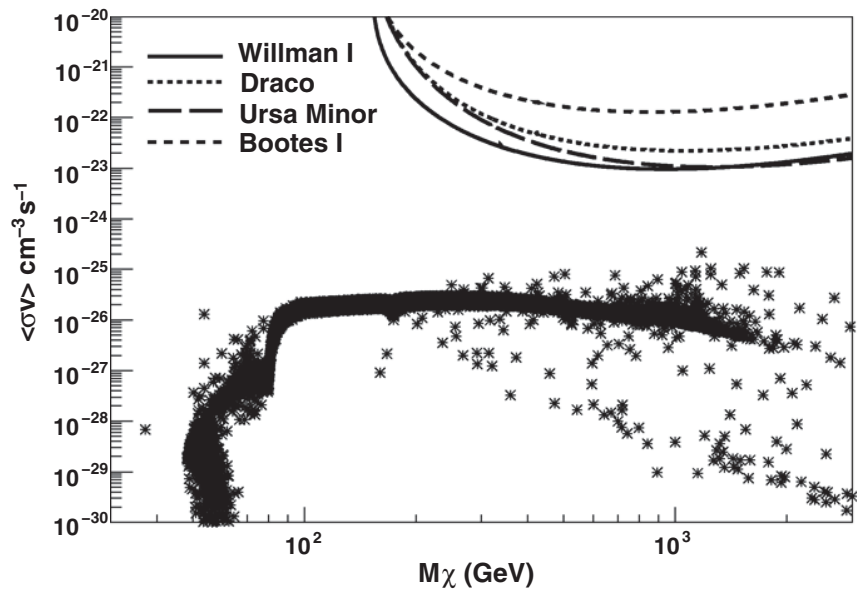

Figure 2. Exclusion regions in the $\left(M_{\chi},\langle\sigma v\rangle\right)$ parameter space based on the results of the observations. It is computed according to Equation (4) using a composite neutralino spectrum (see Wood et al. 2008) and the values of $J$ from Table 1. Black asterisks represent points from MSSM models that fall within \pm 3 standard deviations of the relic density measured in the three-year WMAP data set (Spergel et al. 2007).

hour, significantly constraining upper limits on $\langle\sigma v\rangle$ could be established if $J$ is on the order of $10^{4}$.

Because the factor, $J$, is proportional to the DM density squared, it is subject to considerable uncertainty in its experimental determination. For example, the mass of a DM halo is determined by the interaction of a galaxy with its neighbors and is concentrated in the outer regions of the galaxy. Unlike the DM halo mass, the neutralino annihilation flux is determined by the inner regions of the galaxy where the density is highest. For these regions, the stellar kinematic data do not fully constrain the DM density profile due to limited statistics. Various parameterizations of the DM mass density profile have been put forward (Navarro et al. 1997; Kazantzidis et al. 2004; de Blok et al. 2001; Burkert 1995) based on empirical fits and studies of simulated cold dark matter (CDM) halos. We adopt the assumption of the NFW profile (Navarro et al. 1997) given in Equation (1) which describes a smooth distribution of DM with a single spatial scale factor $r_{s}$. The astrophysical factor, $J$, is then given by

$$
\begin{aligned}
J(\Delta \Omega)= & \left(\frac{2 \pi \rho_{s}^{2}}{\rho_{c}^{2} R_{H}}\right)_{\cos \left(0.115^{\circ}\right)}^{1} \lambda_{\min }\left(\frac{\lambda_{\max }}{r_{s}}\right)^{-2} \\
& \times\left[1+\left(\frac{r(\lambda)}{r_{s}}\right)\right]^{-4} d \lambda d(\cos \theta),
\end{aligned}
$$

where the lower integration bound of 0.115 corresponds to the size of the signal integration region. The galactocentric distance, $r(\lambda)$, is determined by

$$
r(\lambda)=\sqrt{\lambda^{2}+R_{\mathrm{dSph}}^{2}-2 \lambda R_{\mathrm{dSph}} \cos \theta},
$$

where $\lambda$ is the line-of-sight distance and $R_{\mathrm{dSph}}$ is the distance of the dwarf galaxy from the Earth.

Although the integration limits, $\lambda_{\min }$ and $\lambda_{\max }$, are determined by the tidal radius of the $\mathrm{dSph}\left(r_{t}=7 \mathrm{kpc}\right.$ was used for these calculations; Sánchez-Conde et al. 2007), the main contribution to $J(\Delta \Omega)$ comes from the regions $r<r_{s} \ll r_{t}$ and therefore the choice of $r_{t}$ affects the $J$ value negligibly. The main uncertainty for $J$ computation is due to the choice of $\rho_{s}$ and $r_{s}$. For Draco and Ursa Minor, $\rho_{s}$ and $r_{s}$ are taken as the midpoints of the 
range from Strigari et al. (2007). For Willman 1, $\rho_{s}$, and $r_{s}$ are adopted from Bringmann et al. (2009a). The $J$ value Boötes 1 was calculated by Martinez and Bullock as discussed in Section 2. The summary of the $J$ values calculated for each object is given in Table 1 .

An estimated value of $J$ of order 10 is representative for all observed dSphs, which is 3 orders of magnitude below the value needed to constrain generic WIMP models with $m_{\chi} \gtrsim 100 \mathrm{GeV}$ $c^{-2}$. Figure 2 shows the exclusion region in the $\left(m_{\chi},\langle\sigma v\rangle\right)$ parameter space due to the observations reported in this paper. MSSM models shown in the figure were produced with a random scan of the seven-parameter phase space defined in the DarkSUSY package (Gondolo et al. 2004) with the additional WMAP (Spergel et al. 2007) constraint on the cosmological DM energy density.

Several astrophysical factors can increase the value of $J$ as compared to the conservative estimates given in Table 1. First, the inner asymptotic behavior of the DM density may be steeper than $\propto r^{-1}$ predicted by the NFW profile due to unaccounted physical processes at small spatial scales. The extreme assumption would be the Moore profile (Moore et al. 1999) $\propto r^{-1.5}$ asymptotically which generates a logarithmically divergent self-annihilation flux indicating that another physical process, for example self-annihilation, would limit the DM density in the central regions of the galaxy. A second factor that would increase the value of $J$ is deviations of the DM distribution from a smooth average profile (substructures). CDM $N$-body simulations predict substructures in DM halos (Silk \& Stebbins 1993; Diemand et al. 2005, 2007) and the effects on the DM self-annihilation have been studied in these simulations. In general, any regions of DM overdensity will enhance the selfannihilation flux; the cumulative effect of these enhancements is usually referred to as the boost factor. Strigari et al. (2007) find a maximum boost factor of order $10^{2}$ while a more detailed calculation that accounts for the particle properties of the neutralino during formation of DM halos suggests boost factors of order 10 and below (Martinez et al. 2009). Thus, present generation IACTs could be as close as 2 orders of magnitude in sensitivity from constraining generic MSSM models.

Two effects related to the properties of the WIMP particle may improve the chances of the detection of neutralino selfannihilation by ground-based gamma-ray observatories. Internal bremsstrahlung gamma rays produced in neutralino selfannihilation recently calculated by Bringmann et al. (2008) can significantly enhance $d N / d E$ at the energies comparable to $m_{\chi}$ for some MSSM models due to the absence of the helicity suppression factor. Effectively this increases the value of the integral in Equation (4), especially for the higher mass neutralino models. In addition, the $\langle\sigma v\rangle$ for self-annihilation at the present cosmological time may be considerably larger than at the time of WIMP decoupling due to a velocity-dependent term in the cross section and the reduction of the kinetic energy of the WIMP due to the cosmological expansion of the universe (Robertson \& Zentner 2009; Pieri et al. 2009).

\section{CONCLUSIONS}

We have carried out a search for VHE gamma rays from four dSphs: Draco, Ursa Minor, Boötes 1, and Willman 1, as part of an indirect DM search program at the VERITAS observatory. The dSphs were selected for proximity to Earth and for favorable estimates of the $J$ factor based on stellar kinematics data. No significant gamma-ray excess was observed from the four $\mathrm{dSphs}$, and the derived upper limits on the gamma-ray flux constrain the $\langle\sigma v\rangle$ for neutralino pair annihilation as a function of neutralino mass to be $\lesssim 10^{-23} \mathrm{~cm}^{3} \mathrm{~s}^{-1}$ for $m_{\chi} \gtrsim 300 \mathrm{GeV}$ $c^{-2}$. The obtained $\langle\sigma v\rangle$ limits are 3 orders of magnitude above generic predictions for MSSM models assuming an NFW DM density profile, no boost factor, and no additional particle-related gamma-ray flux enhancement factors. Should the neglected effects be included, the constraints on $\langle\sigma v\rangle$ in the most optimistic regime could be pushed to $\lesssim 10^{-25} \mathrm{~cm}^{3} \mathrm{~s}^{-1}$.

To begin confronting the predictions of generic MSSM models through observation of presently known dSphs, future ground-based observatories will need a sensitivity at least an order of magnitude better than present-day instruments. The list of dSphs favorable for observations of DM self-annihilation has grown over the last years by a factor of roughly 2, and it is anticipated that newly discovered $\mathrm{dSphs}$ may offer a larger $J$ factor. The ongoing sky survey conducted by the Fermi Gammaray Space Telescope (FGST) may also identify nearby higher DM density substructures within the MW galaxy which could be followed up by the IACT observatories. Typical current exposures accumulated on dSphs by IACTs are of order $20 \mathrm{hr}$, and ongoing observing programs could feasibly increase the depth of these observations by a factor of 10 (a sensitivity increase of $\sim 3$ ). Improvements in background rejection are anticipated to increase sensitivity by an additional $20 \%-50 \%$. The soon-to-be-operational upgrades, MAGIC-II and HESS-II, as well as a planned VERITAS upgrade will reduce the energy threshold and consequently increase the $d N / d E$ contribution by a factor as large as 10 thus providing an additional sensitivity improvement. With all these factors combined, the $\langle\sigma v\rangle$ limits for $m_{\chi} \gtrsim 300 \mathrm{GeV} c^{-2}$ will begin to rule out the most favorable MSSM models assuming a moderate boost factor. Next generation IACT arrays now being planned such as the Advanced Gamma-ray Imaging System ${ }^{28}$ and the Cherenkov Telescope Array $^{29}$ will provide an order of magnitude increase in sensitivity and lower the energy threshold by factor of $\sim 2$ as compared to VERITAS. These instruments will be able to probe the bulk of the parameter space for generic MSSM models with $m_{\chi} \quad 300 \mathrm{GeV} c^{-2}$ without strong assumptions regarding potential flux enhancement factors.

This research is supported by grants from the US National Science Foundation, the US Department of Energy, and the Smithsonian Institution, by NSERC in Canada, by Science Foundation Ireland, and by STFC in the UK. We acknowledge the excellent work of the technical support staff at the FLWO and the collaborating institutions in the construction and operation of the instrument. V.V.V. acknowledges the support of the U.S. National Science Foundation under CAREER program (Grant No. 0422093).

\section{REFERENCES}

Abdo, A. A., et al. 2010, ApJ, 712, 147

Acciari, V. A., et al. (VERITAS Collaboration) 2008, ApJ, 679, 1427

Aharonian, F., et al. (HESS Collaboration) 2006a, A\&A, 457, 899

Aharonian, F., et al. (HESS Collaboration) 2006b, Phys. Rev. Lett., 97, 221102

Aharonian, F., et al. (HESS Collaboration) 2009, A\&A, 503, 817

Albert, J., et al. (MAGIC Collaboration) 2008, ApJ, 679, 428

Aliu, E., et al. (MAGIC Collaboration) 2009, ApJ, 697, 1299

Aparicio, A., Carrera, R., \& Martínez-Delgado, D. 2001, AJ, 122, 2524

Baltz, E. A., Briot, C., Salati, P., Taillet, R., \& Silk, J. 2000, Phys. Rev. D, 61, 023514

\footnotetext{
28 http://www.agis-observatory.org/

29 http://www.cta-observatory.org/
} 
Bellazzini, M., Ferraro, F. R., Origlia, L., Pancino, E., Monaco, L., \& Olivia, E. 2002, AJ, 124, 3222

Belokurov, V., et al. 2006, ApJ, 647, L11

Belokurov, V., et al. 2007, ApJ, 654, 897

Berge, D., Funk, S., \& Hinton, J. 2007, A\&A, 466, 1219

Bergström, L. 2000, Rep. Prog. Phys., 63, 793

Bergström, L., Ullio, P., \& Buckley, J. 1998, Astropart. Phys., 9, 137

Bertone, G., Fornasa, M., Taoso, M., \& Zentner, A. R. 2009, New J. Phys., 11, 105016

Bertone, G., Hooper, D., \& Silk, J. 2005, Phys. Rep., 405, 279

Bertone, G., Servant, G., \& Sigl, G. 2003, Phys. Rev. D, 68, 044008

Bradač, M., Allen, S. W., Treu, T., Ebeling, H., Massey, R., Morris, R. G., von der Linden, A., \& Applegate, D. 2008, ApJ, 687, 959

Bringmann, T., Bergström, L., \& Edsjö, J. 2008, J. High Energy Phys., JHEP01(2008)049

Bringmann, T., Doro, M., \& Fornasa, M. 2009a, J. Cosmol. Astropart. Phys., JCAP01(2009)016

Bringmann, T., Lavalle, J., \& Salati, P. 2009b, Phys. Rev. Lett., 103, 161301

Bullock, J. S., et al. 2009, arXiv:0902.3492

Burkert, A. 1995, ApJ, 447, L25

Clowe, D., Bradač, M., Gonzalez, A. H., Markevitch, M., Randall, S. W., Jones, C., \& Zaritsky, D. 2006, ApJ, 648, L109

Colafrancesco, S., Profumo, S., \& Ullio, P. 2007, Phys. Rev. D, 75, 023513

de Blok, W. J. G., McGaugh, S. S., Bosma, A., \& Rubin, V. C. 2001, ApJ, 552, L23

Dicus, D. A., Kolb, E. W., \& Teplitz, V. L. 1977, Phys. Rev. Lett., 39, 168

Diemand, J., Kuhlen, M., \& Madau, P. 2007, ApJ, 657, 262

Diemand, J., Moore, B., \& Stadel, J. 2005, Nature, 433, 389

Driscoll, D. D., et al. (STACEE Collaboration) 2008, Phys. Rev. D, 78, 087101

Ellis, J., Hagelin, J. S., Nanopoulos, D. V., Olive, K., \& Srednicki, M. 1984, Nucl. Phys. B, 238, 453

Evans, N. W., Ferrer, F., \& Sarkar, S. 2004, Phys. Rev. D, 69, 123501

Fellhauer, M., Wilkinson, M. I., Evans, N. W., Belokurov, V., Irwin, M. J., Gilmore, G., Zucker, D. B., \& Kleyna, J. T. 2008, MNRAS, 385, 1095

Gondolo, P., Edsjö, J., Ullio, P., Bergström, L., Schelke, M., \& Baltz, E. A. 2004, J. Cosmol. Astropart. Phys., JCAP07(2004)008

Hillas, M. 1985, Proc. 19th ICRC (La Jolla), 445

Irwin, M., \& Hatzidimitriou, D. 1995, MNRAS, 277, 1354

Kazantzidis, S., Mayer, L., Mastropietro, C., Diemand, J., Stadel, J., \& Moore, B. 2004, ApJ, 608, 663

Kleyna, J. T., Wilkinson, M. I., Gilmore, G., \& Evans, N. W. 2003, ApJ, 588, L21

Komatsu, E., et al. 2009, ApJS, 180, 330

Kosack, K., et al. (VERITAS Collaboration) 2004, ApJ, 608, L97
Lee, B. W., \& Weinberg, S. 1977, Phys. Rev. Lett., 39, 165

Li, T-P., \& Ma, Y-Q. 1983, ApJ, 272, 317

Martin, N. F., Ibata, R. A., Chapman, S. C., Irwin, M., \& Lewis, G. F. 2007, MNRAS, 380, 281

Martinez, G. D., Bullock, J. S., Kaplinghat, M., Strigari, L. E., \& Trotta, R. 2009, J. Cosmol. Astropart. Phys., JCAP06(2009)014

Moore, B., Ghigna, S., Governato, F., Lake, G., Quinn, T., Stadel, J., \& Tozzi, P. 1999, ApJ, 524, L19

Muñoz, R. R., Carlin, J. L., Frinchaboy, P. M., Nidever, D. L., Majewski, S. R., \& Patterson, R. J. 2006, ApJ, 650, L5

Muñoz, R. R., et al. 2005, ApJ, 631, L137

Navarro, J. F., Frenk, C. S., \& White, S. D. M. 1997, ApJ, 490, 493

Palma, C., Majewski, S. R., Siegel, M. H., Patterson, R. J., Ostheimer, J. C., \& Link, R. 2003, AJ, 125, 1352

Piatek, S., Pryor, C., Armandroff, T. E., \& Olszewski, E. W. 2002, AJ, 123 , 2511

Pieri, L., Lattanzi, M., \& Silk, J. 2009, MNRAS, 399, 2033

Robertson, B., \& Zentner, A. 2009, Phys. Rev. D, 79, 083525

Rolke, W. A., Lopez, A. M., \& Conrad, J. 2005, Nucl. Instrum. Methods, A551, 493

Sánchez-Conde, M. A., Prada, F., Lokas, E. L., Gomez, M. E., Wojtak, R., \& Moles, M. 2007, Phys. Rev. D, 76, 123509

Servant, G., \& Tait, T. M. P. 2003, Nucl. Phys. B, 650, 391

Shetrone, M. D., Côté, P., \& Sargent, W. L. W. 2001, ApJ, 548, 592

Siegel, M. H., Shetrone, M. D., \& Irwin, M. 2008, AJ, 135, 2084

Silk, J., \& Stebbins, A. 1993, ApJ, 411, 439

Spergel, D. N., et al. 2007, ApJS, 170, 377

Strigari, L. E., Koushiappas, S. M., Bullock, J. S., \& Kaplinghat, M. 2007, Phys. Rev. D, 75, 083526

Strigari, L. E., Koushiappas, S. M., Bullock, J. S., Kaplinghat, M., Simon, J. D., Geha, M., \& Willman, B. 2008, ApJ, 678, 614

Taoso, M., Bertone, G., \& Masiero, A. 2008, J. Cosmol. Astropart. Phys., JCAP03(2008)022

Tyler, C. 2002, Phys. Rev. D, 66, 023509

Walker, M. G., Mateo, M., Olszewski, E. W., Gnedin, O. Y., Wang, X., Bodhisattva, S., \& Woodroofe, M. 2007, ApJ, 667, L53

Walker, M. G., Mateo, M., Olszewski, E. W., Penarrubia, J., Evans, N. W., \& Gilmore, G. 2009, ApJ, 704, 1274

Weekes, T. C., et al. (VERITAS Collaboration) 2002, Astropart. Phys., 17, 221

Wilkinson, M. I., Kleyna, J. T., Evans, N. W., Gilmore, G. F., Irwin, M. J., \& Grebel, E. K. 2004, ApJ, 611, L21

Willman, B., et al. 2005, ApJ, 626, L85

Wood, M., et al. 2008, ApJ, 678, 594

York, D. G., et al. 2000, AJ, 120, 1579 